\title{
Gathering evidence on rare diseases: Anomalous aortic origin of a coronary artery
}

\author{
Marshall L. Jacobs, MD
}

\author{
From the Division of Cardiac Surgery, The Johns Hopkins School of Medicine, Baltimore, Md. \\ Disclosures: Author has nothing to disclose with regard to commercial support. \\ Received for publication Sept 27, 2017; accepted for publication Oct 6, 2017; available ahead of print Nov 9, \\ 2017. \\ Address for reprints: Marshall L. Jacobs, MD, Division of Cardiac Surgery, The Johns Hopkins School of Med- \\ icine, Zayed 7107, 1800 Orleans St, Baltimore, MD 21287 (E-mail: marshall.jacobs@comcast.net). \\ J Thorac Cardiovasc Surg 2018;155:303-4 \\ $0022-5223 / \$ 36.00$ \\ Copyright (C) 2017 Published by Elsevier Inc. on behalf of The American Association for Thoracic Surgery \\ https://doi.org/10.1016/j.jtcvs.2017.10.022
}

The American Association for Thoracic Surgery meeting article titled "Outcomes of Surgical Intervention for Anomalous Aortic Origin of a Coronary Artery: A Large Contemporary Prospective Cohort Study,' by Mery and associates, includes important contributions to a growing body of knowledge concerning a relatively rare, but important congenital anomaly that may account for the uncommon but devastating occurrence of sudden cardiac death in otherwise healthy and active young people. The group at Texas Children's Hospital has organized the evaluation and care of pediatric patients with anomalies of coronary artery origin and course through the establishment of a multidisciplinary team approach that includes institutional protocols for diagnostic evaluation (including imaging and functional studies), handling of management recommendations and patient counseling, surgical management, and evaluation during follow-up. Their approach tacitly accepts the premise that the population of patients is nonhomogeneous in ways that exceed the simple binary classifications of "anomalous left" versus "anomalous right," and "symptomatic versus "asymptomatic." They also acknowledge the existence of gaps in our knowledge of the exact mechanisms that may account for ischemic events in these patients. In this issue of the Journal, they present a detailed review of their experience with 44 patients who underwent an operation for anomalous aortic origin of a coronary artery (AAOCA) as part of the Coronary Anomalies Program at Texas Children's Hospital over a period of just less than 5 years. They emphasize the following key messages:

1. The presentation and anatomy of patients with AAOCA is variable, and as such, a single surgical strategy is likely not ideal for all patients and should be individualized on the basis of anatomy.

2. The decision to recommend surgical intervention should involve both the multidisciplinary team and/or the patient or family.

3. All potential anatomic culprits should be addressed in patients undergoing operation.

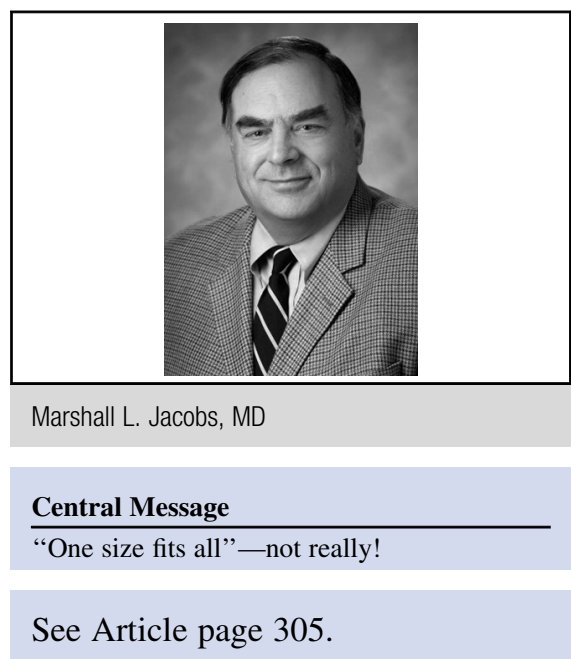

Experience with the management of AAOCA varies considerably across institutions. As recently as 2009, a survey of pediatric cardiologists and heart surgeons found marked heterogeneity in physician opinions regarding AAOCA treatment and management. Since that time, the Congenital Heart Surgeons' Society has developed a Registry of Anomalous Aortic Origin of a Coronary Artery to gather and analyze information on large numbers of young patients with AAOCA for purposes of achieving a better understanding of the natural and surgical history of this anomaly, with the ultimate goal of developing evidence-based treatment and management guidelines. Within the same time frame, the American Heart Association and American College of Cardiology, as well as The American Association for Thoracic Surgery, have put forth updated scientific statements and expert consensus guidelines. However, it is fair to say that in the face of exhaustive research, literature review, evaluation, and classification of available evidence concerning the increased relative risk for sudden death associated with participation in competitive sports in the setting of certain known cardiovascular diseases (including AAOCA), authors of these position articles are generally confounded by the fact that risk-stratifying tools are not available to independently and precisely guide many of these difficult medical decisions. ${ }^{2}$ In a spirit of candor, one would have to say that many, although not absolutely all, of the recommendations and practices adopted by Mery and associates ${ }^{1}$ are harmonized with current guidelines from professional societies. In the face of persisting "knowledge gaps" or deficiencies of available evidence, the approach adopted by 
Mery and colleagues ${ }^{1}$ in Houston seems a reasonable and practical way to try to ensure that each individual patient receives the benefit of currently available knowledge. The approach also emphasizes ongoing analysis of outcomes (both short and intermediate term), which are regularly reviewed with an eye toward revision and refinement of programmatic policies and protocols based on an evolving body of evidence. This is exemplified by the Houston group's evolving approach to functional assessment for inducible ischemia (less reliance on radionuclide stress testing and more frequent performance of stress cardiac magnetic resonance imaging). It is also exemplified by their careful analysis of rare but important unfavorable surgical outcomes. This led to their understanding and elaboration of the potential limitations of unroofing as a surgical technique in the specific setting of an anomalous (wrong sinus) origin of a coronary artery associated with a short intramural course. They point out that this is a circumstance wherein unroofing of the short intramural segment may be technically feasible, but may leave the vessel arising anomalously from the "wrong" sinus of Valsalva with a course behind the intercoronary pillar.

The most recent data analysis from the Society of Thoracic Surgeons Congenital Heart Surgery Database ${ }^{3}$ reveals that 889 patients underwent an operation with primary procedure of "Anomalous aortic origin of coronary artery (AAOCA) repair" over the 4-year period from January 2013 to December 2016. The report includes data from 116 participating centers. Although a sizeable fraction of these 889 total procedures may have been concentrated at specialized centers such as Texas Children's Hospital, it is, nonetheless, an average of only approximately 8 cases per center over the 4-year period of data collection ( 2 cases per center per year). It would not be an easy thing for every center to organize and maintain a multidisciplinary coronary anomalies program. But the Texas Children's group has shown the way for us all to adopt a careful, orderly, and introspective approach to the evaluation and management of these young patients. It is likely, although difficult to be certain, that such an approach will contribute to improved care for the individual patient. Just as important, they offer tools such as the alpha-numeric nomenclature and topography map used to define the site of origin of the coronary arteries during imaging and at surgery. Broader use of such tools will likely help ensure the consistency and quality of the data collected across individual centers and eventually collated and analyzed in multicentric studies, such as those of the Congenital Heart Surgeons' Society Anomalous Aortic Origin of a Coronary Artery Registry.

\section{References}

1. Mery CM, De León LE, Molossi S, Sexson-Tejtel SK, Agrawal H, Krishnamurthy R, et al. Outcomes of surgical intervention for anomalous aortic origin of a coronary artery: a large contemporary prospective cohort study. J Thorac Cardiovasc Surgery. 2018;155:305-19.e4.

2. Maron BJ, Zipes DP, Kovacs RJ. Eligibility and disqualification recommendations for competitive athletes with cardiovascular abnormalities: preamble, principles, and general considerations: a scientific statement from the American Heart Association and American College of Cardiology. Circulation. 2015; 132:e256-61.

3. Jacobs JP, Jacobs ML, Mavroudis C, Tchervenkov CI, Pasquali SK. Executive Summary: The Society of Thoracic Surgeons Congenital Heart Surgery Database Twenty-Sixth Harvest - (January 1, 2013-December 31, 2016). Spring 2017 Harvest. The Society of Thoracic Surgeons (STS and Duke Clinical Research Institute [DCRI]). Durham, NC: Duke University Medical Center; 2017. 